\title{
Classifier of cross talk genes predicts the prognosis of hepatocellular carcinoma
}

\author{
XIAOFENG ZHAI ${ }^{1,2^{*}}$, QINGFENG XUE ${ }^{3 *}$, QUN LIU ${ }^{1,2}$, YUYU GUO ${ }^{1,2}$ and ZHE CHEN ${ }^{1,2}$ \\ ${ }^{1}$ Department of Integrative Oncology, Changhai Hospital of Traditional Chinese Medicine, Second Military Medical \\ University, Shanghai 200433; ${ }^{2}$ Department of Integrative Oncology, Changhai Hospital, Shanghai 200433; ${ }^{3}$ Department \\ of Anesthesiology, Chinese People's Liberation Army 264 Hospital, Taiyuan, Shanxi 030001, P.R. China
}

Received August 6, 2016; Accepted May 5, 2017

DOI: $10.3892 / \mathrm{mmr} .2017 .7003$

\begin{abstract}
The present study aimed to establish a prediction model for hepatocellular carcinoma (HCC) based on the cross talk genes from important biological pathways involved in HCC. Differentially expressed genes (DEGs) for HCC were identified from mRNA profiles of GSE36376, which were mapped to protein-protein interaction (PPI) networks from BioGrid and the human protein reference database. Then critical genes based on the deviation score and the degree of node were selected from the novel PPI network. Cross talk genes were screened from the network established based on the associations of gene-gene, gene-pathway and pathway-pathway. A classifier based on specific cross talk genes was constructed for prediction of HCC using the random forest algorithm. Finally, the diagnostic performance of this prediction model was verified by predicting survival time of patients with HCC from the genome cancer atlas (TCGA) and other independent gene expression omnibus (GEO) databases. From the novel PPI network, a total of 200 critical genes were screened out and they were significantly enriched in 23 pathways, which have been reported to be significantly associated with the development of HCC. Based on these identified pathways, cross talk genes were identified including AKT1, SOS1, EGF, MYC, IGF1, ERBB2, CDKN1B, SHC2, VEGFA and INS. The prediction model has a relative average classification accuracy of 0.94 for $\mathrm{HCC}$, which has a stable predicting efficacy for survival time of HCC patients validated in the TCGA database and two other independent GEO datasets. In conclusion, a total of 39 cross talk genes in HCC were identified and a classifier based on the cross talk genes was constructed, which indicates
\end{abstract}

Correspondence to: Dr Zhe Chen, Department of Integrative Oncology, Changhai Hospital, 168 Changhai Road, Shanghai 200433, P.R. China

E-mail: chendhdhd@163.com

*Contributed equally

Key words: hepatocellular carcinoma, differentially expressed genes, protein-protein interaction network, cross talk genes, pathway a high prognosis prediction efficacy in several independent datasets. The results provide a novel perspective to develop a multiple gene diagnostic tool for HCC prognosis, which also provided potential biomarkers or therapeutic targets for HCC.

\section{Introduction}

Hepatocellular carcinoma (HCC) is one of the most common malignancies, worldwide it is the fifth most common cancer in men and the seventh in women (1). The occurrence and development of HCC is predominantly associated with chronic hepatitis $\mathrm{B}$ virus (HBV) and/or hepatitis $\mathrm{C}$ virus (HCV) infections (2). Surgical resection remains the main therapy for the majority of HCC cases and only 30-40\% of patients with HCC can be cured by surgical resection following diagnosis (3). Exploration of an effective and reliable prediction diagnosis tool for $\mathrm{HCC}$ would markedly improve the prognosis of patients with HCC.

The pathogenesis of HCC has been widely studied. Multiple mechanisms have been reported to be involved in the pathogenesis of HCC including tumor suppressor genes, oncogenes, viral effects and angiogenesis (4). Developing molecular indicators with improved sensitivity and specificity serves an important role in the diagnosis of HCC (5). With the development of high-throughput sequencing technologies, numerous genetic expression profiles associated with tumorigenesis have been established and used for classification and diagnostic prediction of cancer $(6,7)$. However, despite the large quantity of public gene information available, effective diagnostic methods for the prediction of the prognosis of HCC are required.

Genetic mutations are the main factors contributing to tumorigenesis, accompanied by the change of certain critical biological processes including immune regulation, the cell cycle, angiogenesis, wound repair and autophagy (8-12). Differentially expressed genes (DEGs) during tumorigenesis lead to changes in pathways and biological processes. The pathways were not independent in function, however were correlated between the pathways. The cross talk genes shared by the correlated pathways are potential biomarkers and therapeutic targets for cancer. Identification of these cross talk genes may provide important information about HCC.

In the present study, gene expression profile data regarding HCC was downloaded from the public information database, in order to try to establish an effective classifier for HCC 
prognosis prediction based on the cross talk genes involved in HCC. The diagnostic performance of the classifier additionally has been verified in other independent datasets.

\section{Materials and methods}

Data source and preprocessing. The mRNA expression profiles of GSE36376 of HCC were extracted from Gene Expression Omnibus (GEO) (http://www.ncbi.nlm.nih.gov/geo/) using Illumina Human HT-12 v4.0 expression beadchip (GPL10558-11219; Illumina, Inc., HCC, including 240 tumor samples and 193 para-cancerous normal liver tissues. The raw data and probe annotation files were downloaded for analysis.

The probe-level data were obtained from the CEL files and converted into expression value. The data were normalized using the z-score normalization to increase the extent of differential expression. For each sample, the expression values of all probes for a given gene were reduced to a single value by taking the average expression value.

Screening of DEGs. Limma, a microarray analysis program available in the Bioconductor $\mathrm{R}$ package, was used to identify the DEGs in tumor samples compared to the para-cancerous normal liver tissues (13). In order to reduce the information loss caused by multiple-testing adjustment, P-values without adjustment by the false discovery rate were used to identify DEGs. $\mathrm{P}<0.05$ and $\mid \log$ fold chance $(\mathrm{FC}) \mid>1.5$ were set as thresholds to screen out DEGs.

Construction of protein-protein interaction (PPI) network. The human PPIs were downloaded from the Biological General Repository for Interaction Datasets (BioGrid; http://www.thebiogrid.org) and Human Protein Reference Database (HPRD; http://www.hprd.org/). Subsequent to merging two sets of data, a total of 14,553 genes and 662,360 interactions were identified. The DEGs identified above were mapped to the PPI network, in which the non-DEGs that interacted with at least 3 other DEGs were also included. Subsequent to removing the isolated nodes, the PPI network of DEGs associated with HCC was constructed. Subsequently, the network topological properties were analyzed using the network analysis package in Cytoscape software version 3.5.0 (http://www.cytoscape.org/). A total of five key topological indicators were defined to describe the behaviors or characteristics of the nodes in the PPI network, including degrees, average shortest path length (ASPL), closeness centrality (CC), eccentricity (EC) and topological coefficient (TC). Additionally, the hub node was selected based on the degree of the nodes, which was calculated by counting the edges launching from a protein in the PPI network.

Identification of critical genes. Critical genes were selected based on the deviation score of DEGs and the degree of node in the PPI network. The deviation score of DEGs was determined by the expression interval of each gene in para-cancerous normal liver tissue, which was defined as 'I'.

$\mathrm{I}=[\mathrm{min}, \max ]$, where min indicated the subtraction of mean value and standard deviation value of gene ' $i$ ' in para-cancerous normal liver tissue; max indicated the sum of mean value and standard deviation value of gene ' $i$ ' in para-cancerous normal liver tissue.
Deviation score was calculated using Euclidean distance based on the value of gene 'i' expressing in each sample beyond the range of $\mathrm{I}$.

$$
\text { Score }=\sqrt{\sum_{1}^{\mathrm{n}}\left(\mathrm{d}_{\mathrm{i}}-\mathrm{d}\right)^{2}}
$$

Where $d_{i}$ indicated the expression value of gene ' $i$ '. When $\mathrm{d}_{\mathrm{i}}<\min$ in the range of $\mathrm{I}, \mathrm{d}=\min$; when $\mathrm{d}_{\mathrm{i}}>\max$ in the range of $\mathrm{I}$, $\mathrm{d}=\max$. A higher deviation score indicated a bigger deviation of gene ' $\mathrm{i}$ ' in tumor samples compared with that in non-tumor samples.

Critical genes were selected based on the score of $\mathrm{W}$, which was calculated as follows:

$$
\mathrm{W}=\text { Score } * \text { degree }
$$

The degree was normalized using the logarithm at the base of 2. A bigger value of degree indicated more interactions of gene ' $i$ ' interacting with other DEGs and a greater importance. Finally, the $\mathrm{W}$ value of each gene was ranked, and the top 100 genes and the bottom 100 genes were selected as critical genes.

Hierarchical cluster analysis based on changes of pathways. In order to identify the pathways of these critical genes involved, analysis of the top 50 critical genes was conducted using Kyoto encyclopedia of genes and genomes (KEGG) (14) pathways enrichment analysis using the Database for Annotation, Visualization and Integrated Discovery database (15). Enrichment analysis performed on upregulated and downregulated DEGs was determined using a hypergeometric test with $\mathrm{P}<0.1$. The union of pathways from enrichment analysis was defined as the important biological pathways in HCC.

Subsequently, cluster analysis of the samples was performed based on the enriched pathways. The changes of each enriched pathway in each sample were determined by the expression value of DEGs involved in the enriched pathway. The score of indicated pathway was determined by the following formula:

$$
\text { Pathscore }=\log \frac{\sqrt{\sum_{\mathrm{i}}^{\mathrm{m}} \omega\left(\mathrm{d}_{\mathrm{i}}-\overline{\mathrm{d}_{\mathrm{i}}}\right)^{2}}}{\sqrt{\sum_{\mathrm{j}}^{\mathrm{n}} \omega\left(\mathrm{d}_{\mathrm{j}}-\overline{\mathrm{d}_{\mathrm{j}}}\right)^{2}}}
$$

Where pathscore of indicated the score of pathway P; $m$ indicated the number of upregulated DEGs in the enriched pathway of $\mathrm{P} ; \mathrm{n}$ indicated the number of downregulated DEGs in the enriched pathway of $\mathrm{P} ; \overline{\mathrm{d}}_{\mathrm{i}}$ or $\overline{\mathrm{d}}_{\mathrm{i}}$ indicated the average expression value of upregulated gene ' $i$ ' or the downregulated gene ' $\mathrm{j}$ ' in para-cancerous normal liver tissue. Pathscore $>0$ indicated the enriched pathway of $\mathrm{P}$ was upregulated in HCC tissue compared with non-cancer tissue, while a pathscore $<0$ indicated that the pathway of $\mathrm{P}$ was downregulated.

In the end, cluster analysis was performed on all samples and acquired pathways using hierarchical cluster approach (16). Data were pre-processed by logarithmic transformation. All samples and pathways were normalized through median center and similarity matrix was calculated by correlation center. The results were visualized using $\mathrm{R}$ package.

Identification of cross talk genes. Based on the hierarchical cluster results, significant correlations between several important biological pathways were identified. Subsequently, the correlation between the significant pathways was evaluated 


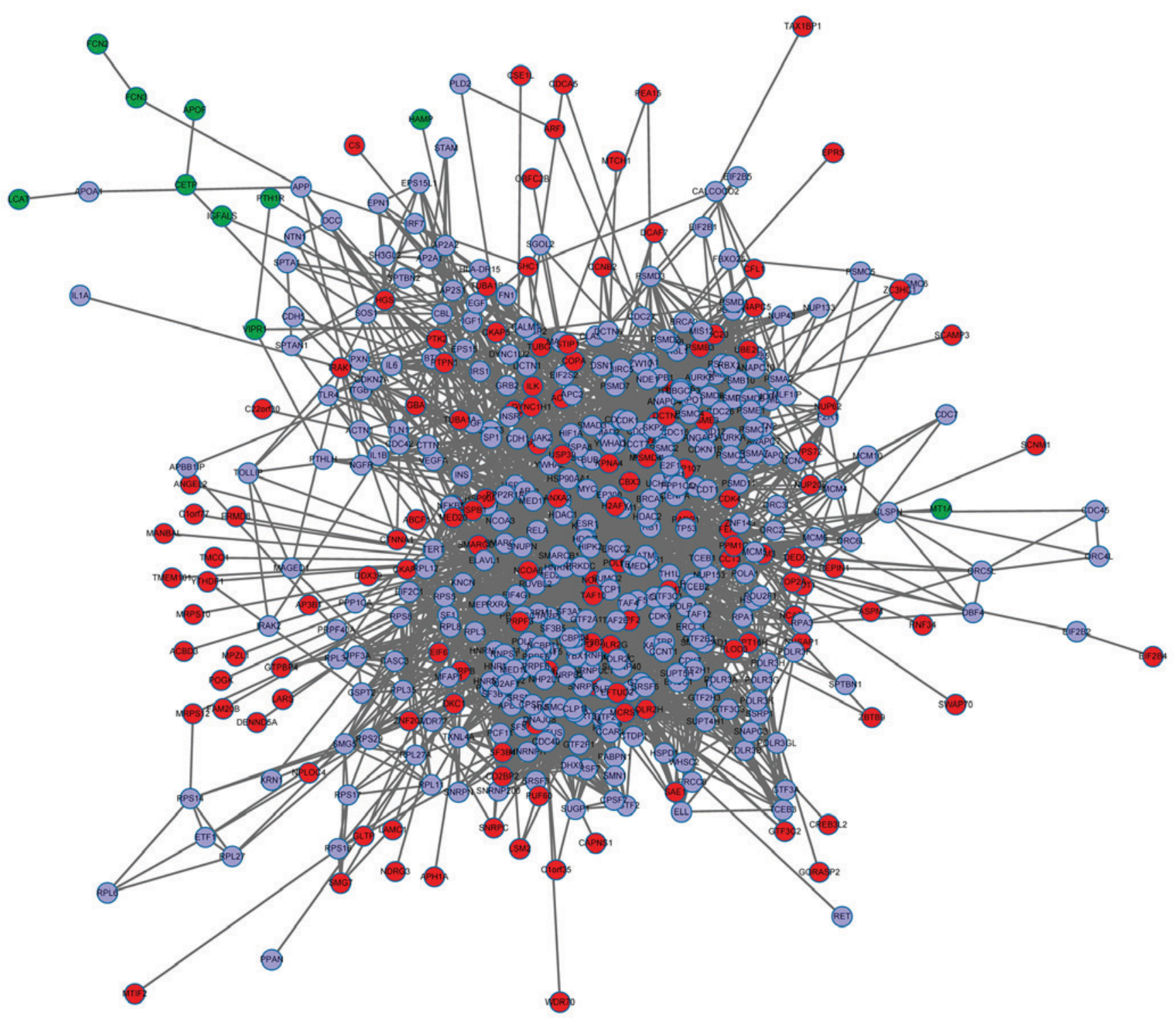

Figure 1. Protein-protein interaction network based on DEGs and non-DEGs interacted with at least 3 DEGs. The red nodes represent the upregulated genes, the green nodes represent the downregulated genes and the blue nodes represent expanded normal genes. DEG, differentially expressed gene.

using Pearson correlation coefficient in the SciPy.stats library within Python (https://docs.scipy.org/doc/scipy/reference/stats. $\mathrm{html}$ ) and identified to be significantly positively or significantly negatively correlated pathways sets. Based on the DEG distribution in each pathway, cross talk genes between correlated pathways were screened out.

Construction of the classifier. A classifier was constructed based on selected cross talk genes from several significantly correlated functional pathways using the random forest algorithm (17). Firstly, all samples were rearranged stochastically and divided into 5 parts: 4 of 5 parts were used as training sets to acquire the threshold parameter for training model, while the other part was used as test set. Following this, the trained classifier was used to predict the accuracy within the test set by calculating the false positive rate and false negative rate. The process was repeated 10 times until all samples regarded as test set were predicted. Receiver operating characteristic (ROC) curve analysis was performed to evaluate the classification performance and robustness of the prediction model.
Validation the prognosis prediction efficacy in other independent datasets. The expression profile information and clinical data of HCC samples was downloaded from The Cancer Genome Atlas (TCGA) and GEO database. Two independent expression profile datasets were downloaded from GEO, which were E-GEOD-54236 and E-GEOD-27150. There were 373 HCC samples obtained from TCGA database, 81 samples in E-GEOD-54236 and 81 samples in E-GEOD-27150. For each sample, abnormal expression of at least one cross talk gene was defined as high risk for HCC, while the patients without cross talk genes changing were considered as low-risk. Next, survival analysis was performed to compare the survival time between high-risk and low-risk patients to validate the prognosis prediction efficacy of identified cross talk genes.

\section{Results}

Identification of DEGs. For the database GSE36376 at $\mathrm{P}=0.05$ and $\log \mathrm{FCl}$ of 1.5 , a total of 249 DEGs were screened between tumor samples and para-cancerous normal liver tissue. Among 
Table I. Key topological indicators of specific genes and all genes in the protein-protein interaction network.

\begin{tabular}{lccc}
\hline Feature & Specific genes & All genes & P-value \\
\hline Degree & 5.23 & 7.01 & $1.46 \times 10^{-2}$ \\
EC & 8.52 & 6.51 & $2.20 \times 10^{-16}$ \\
ASPL & 4.17 & 2.97 & $3.87 \times 10^{-15}$ \\
CC & 0.24 & 0.35 & $2.44 \times 10^{-3}$ \\
TC & 0.24 & 0.17 & $8.40 \times 10^{-9}$ \\
\hline
\end{tabular}

EC, eccentricity; ASPL, average shortest path length; CC, closeness centrality; TC, topological coefficient.

these DEGs, 219 upregulated genes and 30 downregulated genes were included.

Construction of PPInetwork. Firstly, the human protein-protein interactions from the BioGrid and HPRD database were merged, followed by 249 identified DEGs mapped to the PPI network. Subsequent to removing the isolated nodes, the PPI network was constructed containing 504 nodes and 4,650 edges (Fig. 1).

Analysis of network topological properties. The average values of key topological indicators degrees, ASPL, CC, EC and TC were from all genes in the PPI network were calculated and compared between all genes and DEGs. The results were presented in Table I. Compared with those of all genes, DEGs had significantly smaller value of degree and CC, and larger value of EC, ASPL and TC. The changes in these five topological indicators indicated that compared with the background network established based on all genes, the network efficiency of specific network established based on DEGs was reduced: Lower value of degrees indicated lower contribution of each gene to the PPI network; increased values of ASPL, EC and TC, and a decreased value of CC indicated the decreased compactness of the PPI network and decreased capacity for signal transduction between genes.

Identification of critical genes and pathway enrichment. A total of 200 critical genes including the top 100 genes and the bottom 100 genes were screened out. The expression value of these genes deviated significantly in HCC tissues. In addition, these genes are always hub nodes with high degrees in the PPI network (18). Genes that interact with multiple DEGs are possibly involved in regulation of several biological processes $(19,20)$.

KEGG pathway enrichment analysis was performed on 200 critical genes. Following the results, critical genes were identified to be significantly enriched in 23 pathways including pathways in cancer, pathways associated with cell cycle and cell apoptosis.

The abnormal expression of upregulated or downregulated genes leads to an imbalance of the pathway. The extent of this imbalance is determined by the extent of deviation of all upregulated or downregulated genes involved in the pathway. As presented in Fig. 2, the HCC tissue samples could be effectively distinguished from normal liver tissue based on the pathscore of the identified 23 pathways. The correlation between the pathways was evaluated based on the pathscore using Pearson correlation coefficient in SciPy.stats library within Python. When the correlation coefficient $-0.5<\mathrm{r}>0.5$, two pathways were identified as significantly correlated with each other. Based on the value of $r$, a heat map was plotted indicating the correlation between pathways (Fig. 3).

Identification of cross talk genes. To identify cross talk genes, a network based on the gene-gene, gene-pathway and pathway-pathway associations was established (Fig. 4). The network included 61 nodes and 367 edges. Total of 22 nodes were pathways and the remaining 39 nodes were genes.

As presented in Fig. 4, there may be more than one cross talk gene between two pathways. For example, there are seven cross talk genes between hsa05200: Pathways in cancer and hsa05213: Endometrial cancer, including ERBB2, AXIN1, SOS1, AKT1, APC2, MYC and EGF. A cross talk gene could be shared with more than two pathways, which indicated the cross talk gene participated in multiple important biological pathways, suggesting the potential biomarker or therapeutic targets. The top 10 cross talk genes involved in regulating the maximum of pathways were listed in Table II.

Construction of classifier. The classification performance and robustness of the classfier were evaluated by ROC analysis. The results are presented in Fig. 5, which showed high classification performance with the lowest accuracy 0.91 and the average accuracy 0.94 .

Three-dimension coordinate system was established and three genes with top contribution were set as coordinate axis (Fig. 6). Samples from HCC tissue or normal liver tissue were distinguished with different colors.

Validation of the prognosis prediction efficacy in other independent datasets. In the present study, the survival time of patients with HCC was verified using the TCGA dataset to evaluate the prediction effects of the classifier. Patients with at least one differentially expressed cross talk gene were considered high-risk cases, while patients without differentially expressed cross talk gene were low-risk cases. Subsequently, the survival time between high-risk and low-risk cases was compared. The results were presented in Fig. 7. There was a significant difference of survival time $(\mathrm{P}=0.011)$, indicating the high sensitivity and stability of the prediction model established in the present study.

Another two datasets; E-GEOD-54236 and E-GEOD-27150; were also downloaded and used to compare the survival time of patients with HCC. The results were displayed in Figs. 8 and 9. There an additional significant difference in the total survival time $(\mathrm{P}<0.05)$ between highand low-risk cases. These results indicated that the identified cross talk genes indicated a relatively poor prognosis in HCC.

\section{Discussion}

Discovery of biomarkers associated with HCC contributes to diagnosis and treatment of $\mathrm{HCC}$, which is beneficial for the 


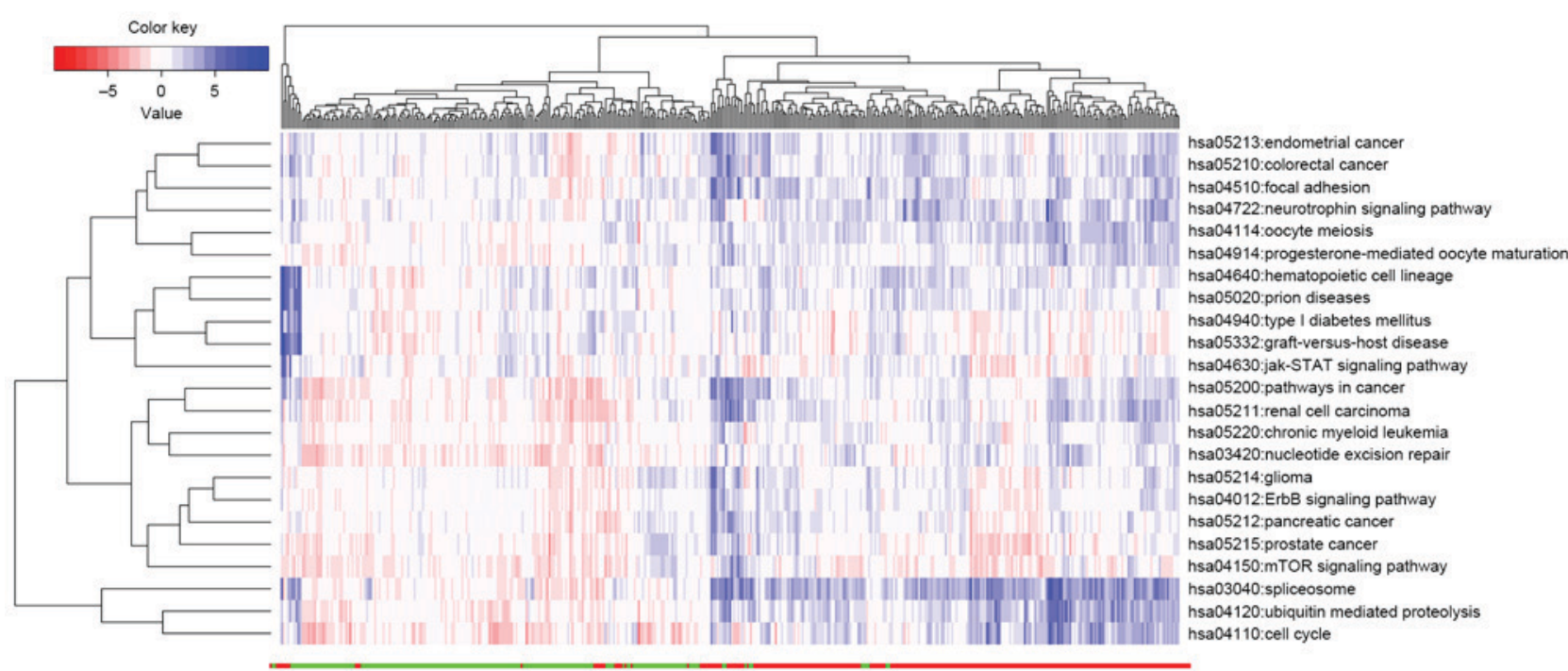

Figure 2. Heatmap for hierarchical cluster analysis. The horizontal axis represents samples of hepatocellular carcinoma and para-cancerous normal liver tissue. The vertical axis represents 23 identified pathways. The heat map indicates the deviation score of each pathways in any sample. The blue region represents the downregulated pathways and the red region represents the upregulated pathways.

\begin{tabular}{|c|c|c|c|c|c|c|c|c|c|c|c|c|c|c|c|c|c|c|c|c|c|c|c|}
\hline & & $\neg$ & $\Gamma$ & $\neg$ & & 7 & & $\square$ & & & $=$ & & & & & & $\longrightarrow$ & $\Gamma$ & ᄀ & $\Gamma$ & $\Gamma$ & & \\
\hline 1.00 & 0.27 & 0.33 & 0.44 & 0.26 & 0.26 & 0.23 & -0.09 & -0.07 & -0.11 & -0.11 & -0.18 & -0.15 & -0.09 & 0.20 & 0.17 & 0.26 & 0.28 & 0.20 & 0.30 & 0.15 & 0.04 & 0.14 & 0.8 \\
\hline 0.27 & 1.00 & 0.73 & 0.66 & 0.50 & 0.20 & 0.27 & 0.25 & 0.21 & 0.09 & 0.14 & 0.33 & 0.26 & -0.02 & -0.11 & 0.10 & 0.01 & 0.10 & 0.33 & 0.26 & 0.22 & 0.21 & 0.35 & 0.6 \\
\hline 0.33 & 0.73 & 1.00 & 0.74 & 0.55 & 0.14 & 0.23 & 0.25 & 0.23 & 0.03 & 0.17 & 0.22 & 0.17 & -0.12 & -0.08 & 0.04 & -0.04 & 0.07 & 0.19 & 0.17 & 0.23 & 0.07 & 0.25 & 0.4 \\
\hline 0.44 & 0.66 & 0.74 & 1.00 & 0.83 & -0.03 & -0.00 & -0.01 & -0.07 & -0.16 & -0.04 & -0.07 & -0.10 & -0.06 & -0.09 & -0.00 & -0.07 & -0.03 & 0.06 & 0.05 & -0.04 & -0.08 & 0.10 & .2 \\
\hline 0.26 & 0.50 & 0.55 & 0.83 & 1.00 & -0.07 & -0.07 & -0.09 & 0.00 & -0.02 & -0.07 & -0.02 & -0.09 & 0.15 & 0.08 & 0.04 & -0.04 & -0.03 & 0.09 & 0.07 & -0.02 & -0.02 & 0.07 & \\
\hline 0.26 & 0.20 & 0.14 & -0.03 & -0.07 & 1.00 & 0.59 & 0.54 & 0.35 & 0.39 & 0.51 & 0.52 & 0.44 & 0.18 & 0.32 & 0.31 & 0.58 & 0.76 & 0.47 & 0.40 & 0.50 & 0.58 & 0.56 & \\
\hline 0.23 & 0.27 & 0.23 & -0.00 & -0.07 & 0.59 & 1.00 & 0.42 & 0.47 & 0.32 & 0.24 & 0.52 & 0.43 & -0.12 & 0.02 & 0.13 & 0.33 & 0.41 & 0.47 & 0.39 & 0.46 & 0.39 & 0.28 & \\
\hline-0.09 & 0.25 & 0.25 & -0.01 & -0.09 & 0.54 & 0.42 & 1.00 & 0.74 & 0.57 & 0.70 & 0.68 & 0.81 & -0.05 & -0.08 & 0.03 & -0.01 & 0.24 & 0.32 & 0.14 & 0.26 & 0.46 & 0.47 & \\
\hline-0.07 & 0.21 & 0.23 & -0.07 & 0.00 & 0.35 & 0.47 & 0.74 & 1.00 & 0.73 & 0.49 & 0.66 & 0.75 & 0.04 & 0.07 & 0.06 & 0.04 & 0.16 & 0.35 & 0.20 & 0.41 & 0.39 & 0.40 & \\
\hline-0.11 & 0.09 & 0.03 & -0.16 & -0.02 & 0.39 & 0.32 & 0.57 & 0.73 & 1.00 & 0.47 & 0.61 & 0.73 & 0.46 & 0.37 & 0.36 & 0.31 & 0.36 & 0.36 & 0.26 & 0.52 & 0.59 & 0.54 & \\
\hline-0.11 & 0.14 & 0.17 & -0.04 & -0.07 & 0.51 & 0.24 & 0.70 & 0.49 & 0.47 & 1.00 & 0.64 & 0.62 & 0.23 & 0.18 & 0.21 & 0.20 & 0.36 & 0.25 & 0.14 & 0.37 & 0.55 & 0.57 & \\
\hline-0.18 & 0.33 & 0.22 & -0.07 & -0.02 & 0.52 & 0.52 & 0.68 & 0.66 & 0.61 & 0.64 & 1.00 & 0.81 & 0.18 & 0.06 & 0.28 & 0.19 & 0.37 & 0.55 & 0.39 & 0.51 & 0.70 & 0.60 & \\
\hline-0.15 & 0.26 & 0.17 & -0.10 & -0.09 & 0.44 & 0.43 & 0.81 & 0.75 & 0.73 & 0.62 & 0.81 & 1.00 & 0.10 & 0.00 & 0.20 & 0.08 & 0.27 & 0.44 & 0.26 & 0.43 & 0.69 & 0.56 & \\
\hline-0.09 & -0.02 & -0.12 & -0.06 & 0.15 & 0.18 & -0.12 & -0.05 & 0.04 & 0.46 & 0.23 & 0.18 & 0.10 & 1.00 & 0.61 & 0.62 & 0.43 & 0.35 & 0.14 & 0.14 & 0.41 & 0.56 & 0.51 & \\
\hline 0.20 & -0.11 & -0.08 & -0.09 & 0.08 & 0.32 & 0.02 & -0.08 & 0.07 & 0.37 & 0.18 & 0.06 & 0.00 & 0.61 & 1.00 & 0.66 & 0.74 & 0.69 & 0.18 & 0.43 & 0.53 & 0.41 & 0.53 & \\
\hline 0.17 & 0.10 & 0.04 & -0.00 & 0.04 & 0.31 & 0.13 & 0.03 & 0.06 & 0.36 & 0.21 & 0.28 & 0.20 & 0.62 & 0.66 & 1.00 & 0.65 & 0.70 & 0.35 & 0.57 & 0.67 & 0.61 & 0.65 & \\
\hline 0.26 & 0.01 & -0.04 & -0.07 & -0.04 & 0.58 & 0.33 & -0.01 & 0.04 & 0.31 & 0.20 & 0.19 & 0.08 & 0.43 & 0.74 & 0.65 & 1.00 & 0.82 & 0.37 & 0.56 & 0.67 & 0.48 & 0.54 & \\
\hline 0.28 & 0.10 & 0.07 & -0.03 & -0.03 & 0.76 & 0.41 & 0.24 & 0.16 & 0.36 & 0.36 & 0.37 & 0.27 & 0.35 & 0.69 & 0.70 & 0.82 & 1.00 & 0.47 & 0.68 & 0.67 & 0.57 & 0.66 & \\
\hline 0.20 & 0.33 & 0.19 & 0.06 & 0.09 & 0.47 & 0.47 & 0.32 & 0.35 & 0.36 & 0.25 & 0.55 & 0.44 & 0.14 & 0.18 & 0.35 & 0.37 & 0.47 & 1.00 & 0.78 & 0.46 & 0.56 & 0.62 & \\
\hline 0.30 & 0.26 & 0.17 & 0.05 & 0.07 & 0.40 & 0.39 & 0.14 & 0.20 & 0.26 & 0.14 & 0.39 & 0.26 & 0.14 & 0.43 & 0.57 & 0.56 & 0.68 & 0.78 & 1.00 & 0.56 & 0.47 & 0.59 & \\
\hline 0.15 & 0.22 & 0.23 & -0.04 & -0.02 & 0.50 & 0.46 & 0.26 & 0.41 & 0.52 & 0.37 & 0.51 & 0.43 & 0.41 & 0.53 & 0.67 & 0.67 & 0.67 & 0.46 & 0.56 & 1.00 & 0.62 & 0.66 & \\
\hline 0.04 & 0.21 & 0.07 & -0.08 & -0.02 & 0.58 & 0.39 & 0.46 & 0.39 & 0.59 & 0.55 & 0.70 & 0.69 & 0.56 & 0.41 & 0.61 & 0.48 & 0.57 & 0.56 & 0.47 & 0.62 & 1.00 & 0.79 & \\
\hline 0.14 & 0.35 & 0.25 & 0.10 & 0.07 & 0.56 & 0.28 & 0.47 & 0.40 & 0.54 & 0.57 & 0.60 & 0.56 & 0.51 & 0.53 & 0.65 & 0.54 & 0.66 & 0.62 & 0.59 & 0.66 & 0.79 & 1.00 & \\
\hline 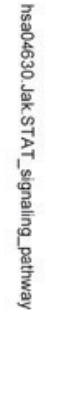 & 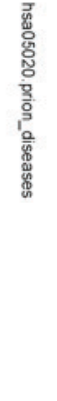 & 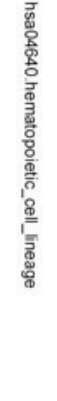 & 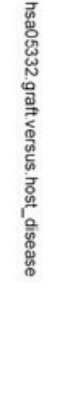 & 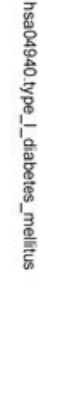 & 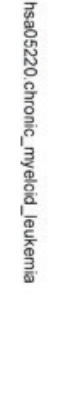 & 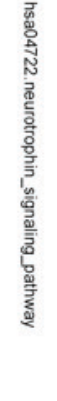 & 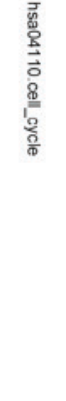 & 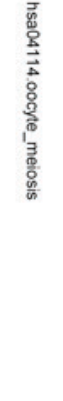 & 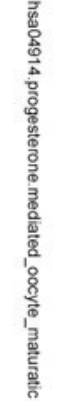 & 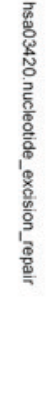 & 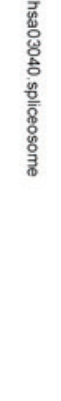 & 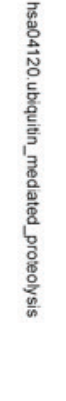 & 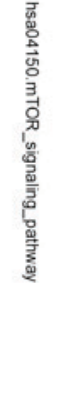 & 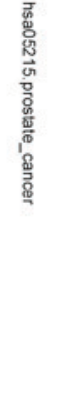 & 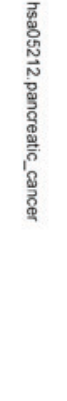 & 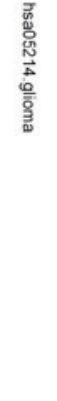 & 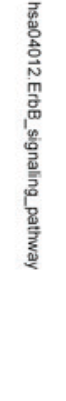 & 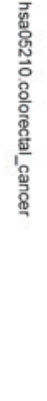 & 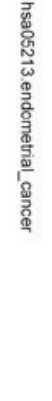 & 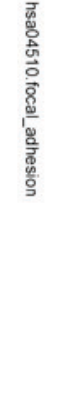 & 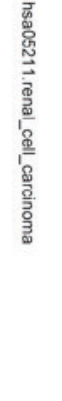 & 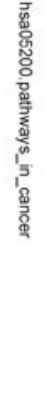 & \\
\hline
\end{tabular}

Figure 3. Heatmap indicating the correlation between 23 pathways. The yellow or orange boxes represent a close association between the pathways. 


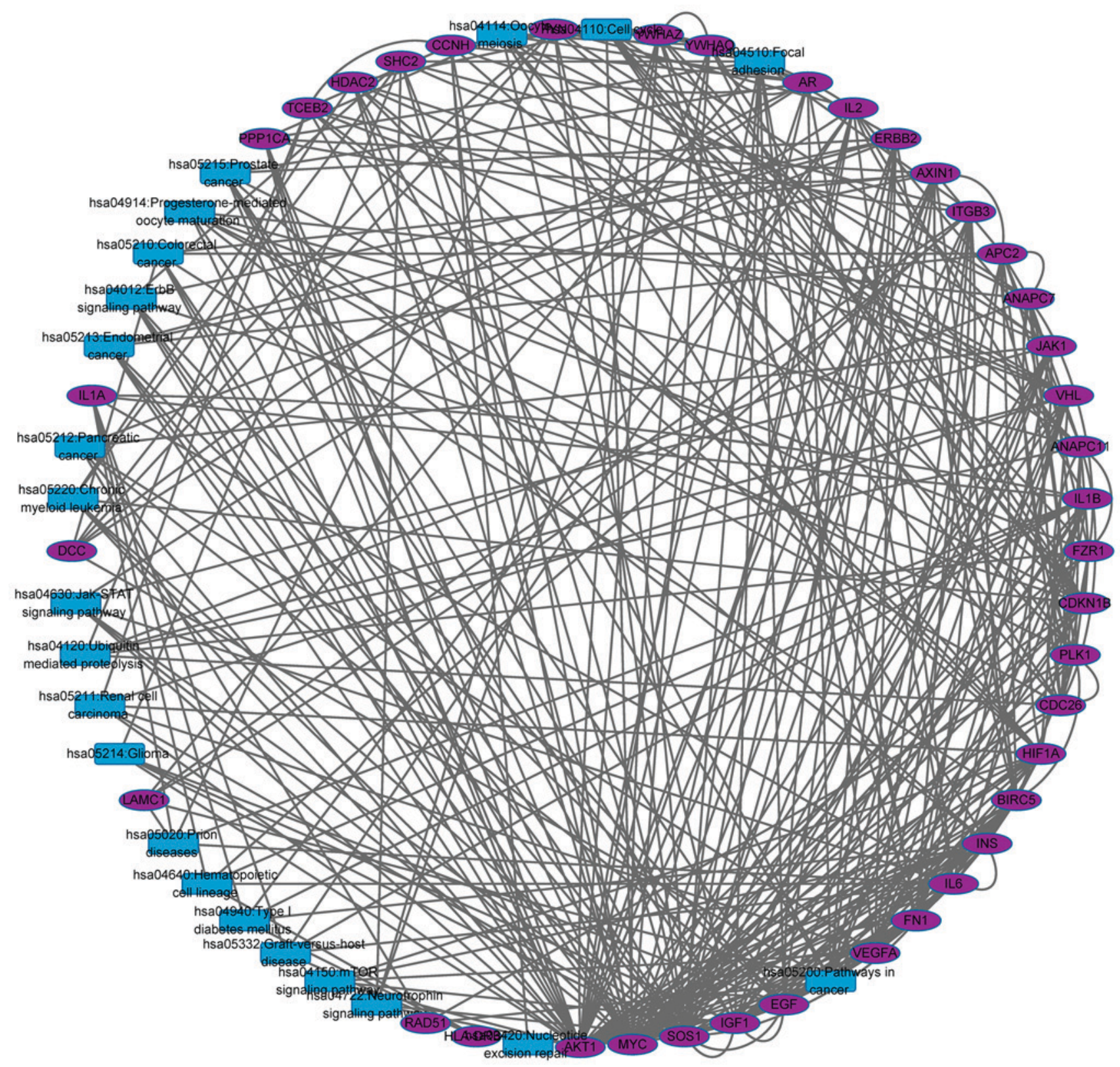

Figure 4. A network established based the associations between identified genes and genes, identified genes and identified pathways, and identified pathways and pathways. The blue boxes represent identified pathways and purple oval boxes represent identified genes. The edges represents the gene-gene and gene-pathway interactions.

improvement of prognosis in patients with HCC (21). Multiple genetic alternations have been identified in HCC. These changes may be regulated by global regulatory mechanisms that control co-operation among various metabolic and signaling pathways (22). Thus the cross talk genes screened from these correlated pathways are important biomarkers for HCC.

In the present study, the mRNA expression profile of GSE36376 was downloaded and DEGs were screened. A total of 219 upregulated genes and 30 downregulated genes were obtained between tumor samples and para-cancerous normal liver tissue, which were mapped to the merged PPI network. Subsequently, the critical genes were selected based on the deviation score of DEGs and the degree of node in the PPI network (23). A total of 200 critical genes were screened out. KEGG pathway enrichment analysis indicated that these critical genes were significantly enriched in 23 pathways including pathways in cancer, pathways associated with cell cycle and cell apoptosis, which have been reported to be significantly associated with the development of $\operatorname{HCC}(10,24)$. Next, the interactions between pathways were identified by hierarchical cluster analysis based on the deviation score. The results indicated that the deviation score of 23 identified pathways distinguished the HCC tissue samples from normal liver tissue effectively.

The cross talk genes are interpreted as genes co-existing in two or more pathways and connecting several biological pathways. The abnormal expression of cross talk genes lead to a similar trend of changes between pathways that are regulated by them. To identify these cross talk genes, a network based on the gene-gene, gene-pathway and pathway-pathway associations was constructed. A total of 39 genes were identified as cross talk genes, in which the top 10 cross talk genes were protein kinase $\mathrm{B} \alpha$ (AKT1), son of sevenless homolog 1 (SOS1), epidermal 
Table II. Top 10 cross talk genes identified and number of involved pathways.

Cross talk gene

Pathway number

\begin{tabular}{lc}
\hline AKT1 & 14 \\
SOS1 & 11 \\
EGF & 7 \\
MYC & 7 \\
IGF1 & 7 \\
ERBB2 & 6 \\
CDKN1B & 5 \\
SHC2 & 5 \\
VEGFA & 5 \\
INS & 5 \\
\hline
\end{tabular}

AKT1, protein kinase B $\alpha$; SOS1, son of sevenless homolog 1; EGF, epidermal growth factor; IGF1, insulin like growth factor 1; ERBB2, Erb-B2 receptor tyrosine kinase 2; CDKN1B, cyclin dependent kinase inhibitor 1B; SHC2, SHC adaptor protein 2; VEGFA, vascular endothelial growth factor A; INS, insulin.

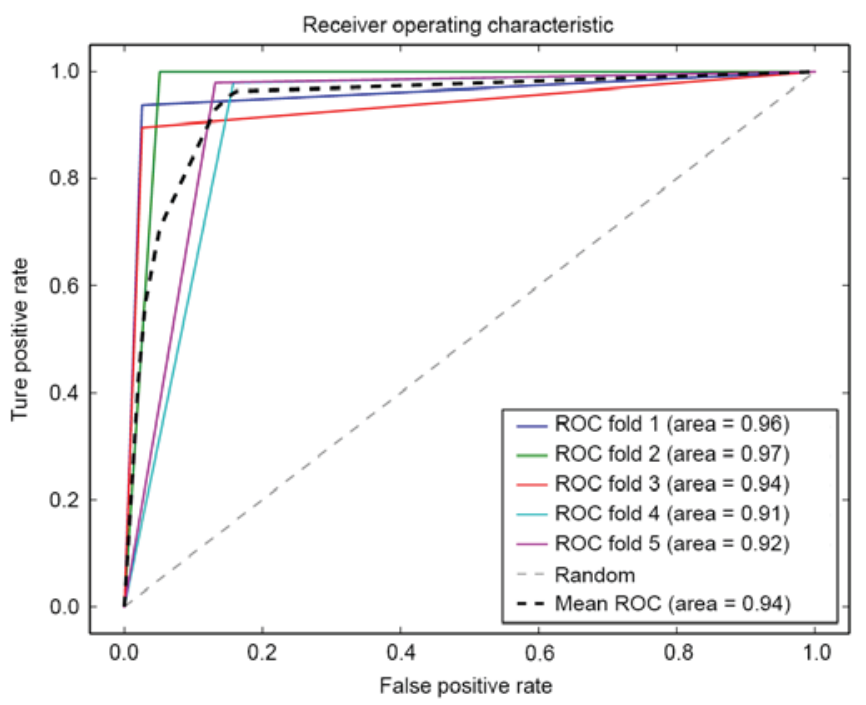

Figure 5. ROC curves for the evaluation of classification performance. ROC, receiver operating characteristic.

growth factor (EGF), MYC, insulin like growth factor 1 (IGF1), Erb-B2 receptor tyrosine kinase 2 (ERBB2), cyclin dependent kinase inhibitor 1B (CDKN1B), SHC adaptor protein 2 (SHC2), vascular endothelial growth factor A (VEGFA) and insulin (INS). All these identified genes were significantly correlated with the occurrence and development of HCC.

AKT1 is one of the most important members of the AKT family, in which phosphorylation has been indicated as a risk factor for early disease recurrence and poor prognosis in HCC (25). EGF is an important mitogen for hepatocytes and its overexpression has been demonstrated to promote HCC (26). In addition, targeting the EGF receptor has been observed to be an effective therapy for treating HCC (27). A previous study indicated that inactivation of the MYC oncogene is sufficient to induce sustained regression of invasive liver cancer including

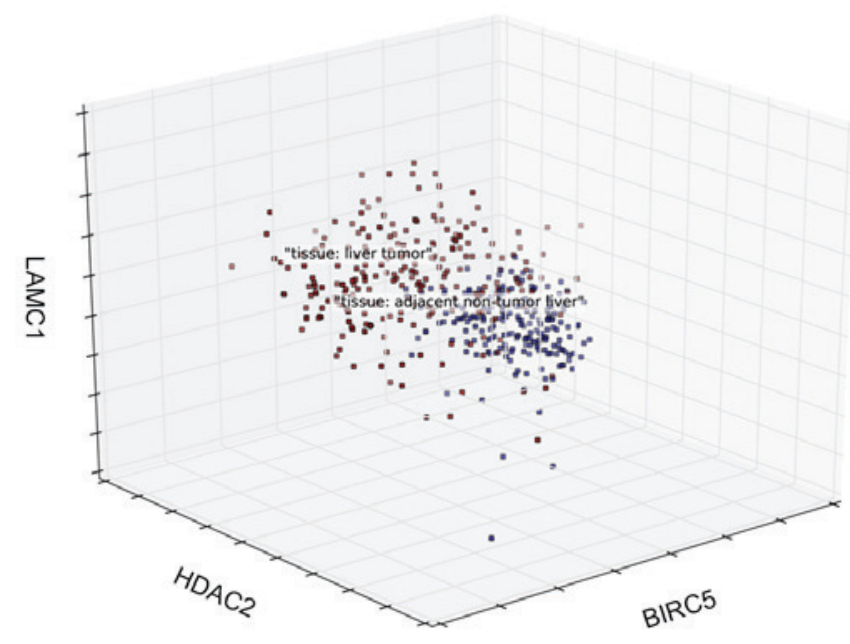

Figure 6. Three-dimensional coordinate system to directly indicate the sample distribution based on the top three cross talk genes.

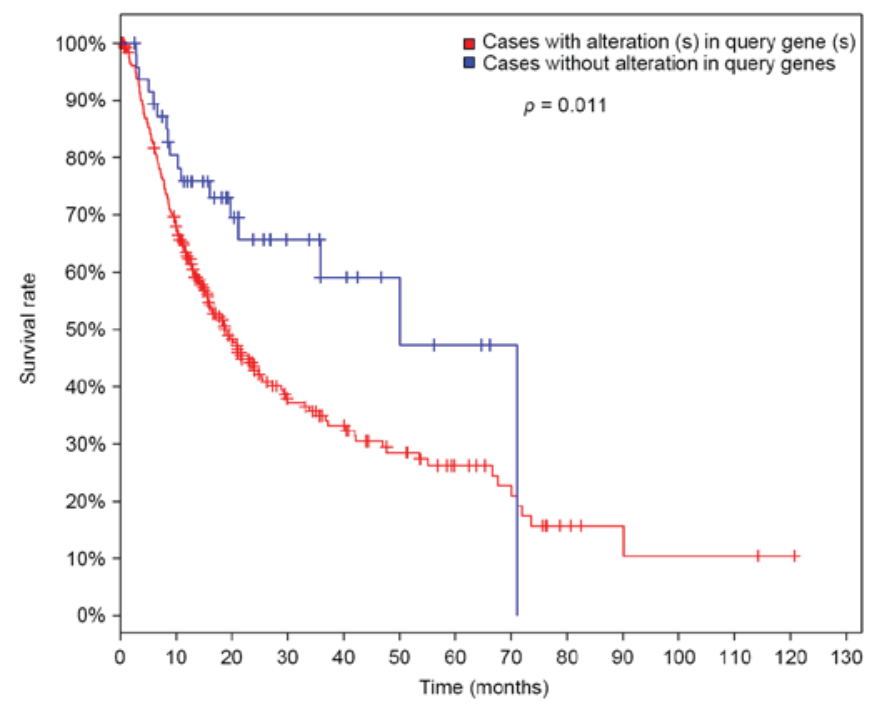

Figure 7. Survival analysis using The Cancer Genome Atlas database to validate cross talk genes. The blue plot represents cases at low risk for HCC and the red plot represents cases at high risk for HCC. HCC, hepatocellular carcinoma.

uncovering the pluripotent capacity of tumors to differentiate into normal cellular lineages and inducing tumors to a state of tumor dormancy (28). Previous studies have indicated IGF1 is a promising biomarker for detection of early HCC (29) and blockage of IGF signaling has been used in HCC treatment in clinical trials (30). ERBB2, a member of the epidermal growth factor receptor family, has been indicated to be expressed in a significant number of hepatoma cancer types including $\mathrm{HCC}$, acting as an independent prognostic factor and a major contributor to carcinogenesis (31). CDKN1B has been reported to a direct target of miR-22, and downregulation of CDKN1B by miR-22 can promote growth of HCC cells and affect HCC prognosis (32). SHC2 has been reported to be an important molecule in cellular signaling pathways in the pathogenesis of HCC (33). VEGFA, a member of the VEGF family, is one of the most potent angiogenic factors expressed in various types of human cancer including HCC (34). The vascular invasion 


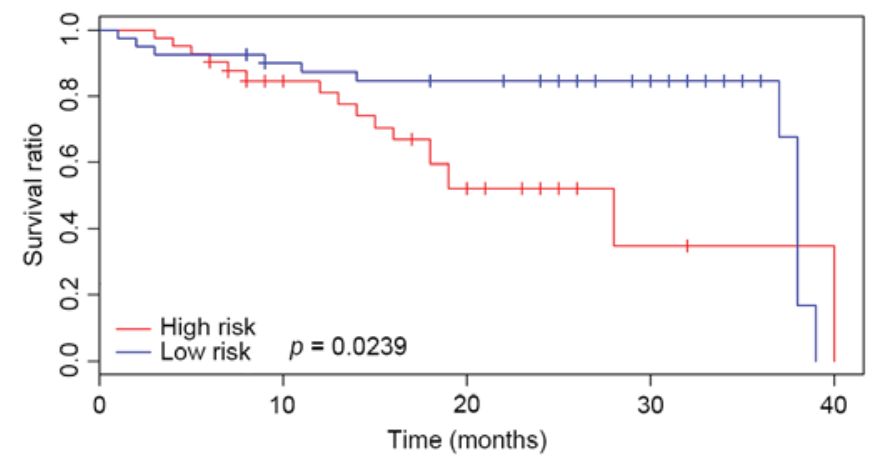

Figure 8. Survival analysis using E-GEOD-27,150 database to validate cross talk genes. The blue plot represents cases at low risk for HCC and the red plot represents cases at high risk for HCC. HCC, hepatocellular carcinoma.

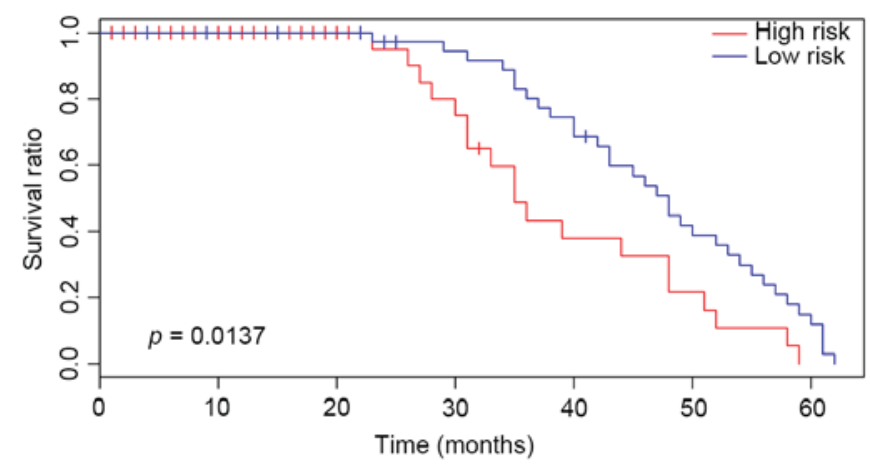

Figure 9. Survival analysis using E-GEOD-54,236 database to validate cross talk genes. The blue plot represents cases at low risk for HCC and the red plot represents cases at high risk for HCC. HCC, hepatocellular carcinoma.

and metastasis of HCC is always associated with the expression of VEGFA (35). Additionally, INS polymorphisms have been observed to be associated with cancer risk including that of HCC (36). No direct evidence has indicated association of SOS1 with HCC, while enhanced expression of SOS1 has been observed in several other types of cancer $(37,38)$.

The identified cross talk genes were used as a classifier to classify the HCC samples from normal tissue, which was observed to exhibit high accuracy with the lowest accuracy as 0.91 , and an average accuracy of 0.94 . Finally, the prognosis prediction effects of the classifier were validated in TCGA and two other independent GEO datasets. The results indicate a high sensitivity and stability of the prognosis prediction efficacy for patients with HCC.

In conclusion, the current study identified 39 cross talk genes of HCC and a classifier based on the cross talk genes was constructed, which exhibited high prognosis prediction efficacy in several independent datasets. The results provide a novel perspective to develop a multiple gene diagnostic tool for HCC prognosis, which also provide potential biomarkers or therapeutic targets for HCC.

\section{Acknowledgements}

The present study was supported by Shanghai Municipal Commission of Health and Family Planning (grant no. ZYSNXD-CC-ZDYJ032).

\section{References}

1. Torre LA, Bray F, Siegel RL, Ferlay J, Lortet-Tieulent J and Jemal A: Global cancer statistics, 2012. CA Cancer J Clin 65: 87-108, 2015

2. Perz JF, Armstrong GL, Farrington LA, Hutin YJ and Bell BP: The contributions of hepatitis $B$ virus and hepatitis $C$ virus infections to cirrhosis and primary liver cancer worldwide. J Hepatol 45: 529-538, 2006.

3. Marrero JA, Kudo M and Bronowicki JP: The challenge of prognosis and staging for hepatocellular carcinoma. Oncologist 15 (Suppl 4): S23-S33, 2010.

4. Farazi PA and DePinho RA: Hepatocellular carcinoma pathogenesis: From genes to environment. Nat Rev Cancer 6: 674-687, 2006.

5. Li D and Satomura S: Biomarkers for hepatocellular carcinoma (HCC): An update. Adv Exp Med Biol 867: 179-193, 2015.

6. Reuter JA, Spacek DV and Snyder MP: High-throughput sequencing technologies. Mol Cell 58: 586-597, 2015.

7. Schmidt D, Wilson MD, Spyrou C, Brown GD, Hadfield J and Odom DT: ChIP-seq: Using high-throughput sequencing to discover protein-DNA interactions. Methods 48: 240-248, 2009.

8. Mathew R, Karantza-Wadsworth V and White E: Role of autophagy in cancer. Nat Rev Cancer 7: 961-967, 2007.

9. Astolfi A, Landuzzi L, Nicoletti G, De Giovanni C, Croci S, Palladini A, Ferrini S, Iezzi M, Musiani P, Cavallo F, et al: Gene expression analysis of immune-mediated arrest of tumorigenesis in a transgenic mouse model of HER-2/neu-positive basal-like mammary carcinoma. Am J Pathol 166: 1205-1216, 2005.

10. Kamb A, Gruis NA, Weaver-Feldhaus J, Liu Q, Harshman K, Tavtigian SV, Stockert E, Day RS III, Johnson BE and Skolnick MH: A cell cycle regulator potentially involved in genesis of many tumor types. Science 264: 436-440, 1994.

11. Bergers $\mathrm{G}$ and Benjamin LE: Tumorigenesis and the angiogenic switch. Nat Rev Cancer 3: 401-410, 2003.

12. Arwert EN, Hoste E and Watt FM: Epithelial stem cells, wound healing and cancer. Nat Rev Cancer 12: 170-180, 2012.

13. Berkeley C: Linear models and empirical Bayes methods for assessing differential expression in microarray experiments. Stat Appl Genet Mol Biol 3: Article3, 2004.

14. Aoki-Kinoshita K, Kanehisa M and Bergman N: Comparative genomics. Journal, 2007.

15. Huang DW, Sherman BT, Tan Q, Collins JR, Alvord WG, Roayaei J, Stephens R, Baseler MW, Lane HC and Lempicki RA: The DAVID gene functional classification tool: A novel biological module-centric algorithm to functionally analyze large gene lists. Genome Biol 8: R183, 2007.

16. Köhn HF and Hubert LJ: Hierarchical cluster analysis. Wiley StatsRef: Statistics Reference Online, 2006.

17. Liaw A and Wiener M: Classification and regression by randomForest. R News 2: 18-22, 2002.

18. Vazquez A, Flammini A, Maritan A and Vespignani A: Global protein function prediction from protein-protein interaction networks. Nat Biotechnol 21: 697-700, 2003.

19. Colcombet J and Hirt H: Arabidopsis MAPKs: A complex signalling network involved in multiple biological processes. Biochem J 413: 217-226, 2008.

20. Stuart JM, Segal E, Koller D and Kim SK: A gene-coexpression network for global discovery of conserved genetic modules. Science 302: 249-255, 2003.

21. Zinkin NT, Grall F, Bhaskar K, Otu HH, Spentzos D, Kalmowitz B, Wells M, Guerrero M, Asara JM, Libermann TA and Afdhal NH: Serum proteomics and biomarkers in hepatocellular carcinoma and chronic liver disease. Clin Cancer Res 14: 470-477, 2008.

22. Lodish H, Berk A, Kaiser CA, Krieger M, Bretscher A, Ploegh H and Amon A: Molecular Cell Biology. 7th edition. W H Freeman and Company, New York, 2012.

23. Przulj N, Wigle DA and Jurisica I: Functional topology in a network of protein interactions. Bioinformatics 20: 340-348, 2004.

24. Fabregat I: Dysregulation of apoptosis in hepatocellular carcinoma cells. World J Gastroenterol 15: 513-520, 2009.

25. Nakanishi K, Sakamoto M, Yamasaki S, Todo S and Hirohashi S: Akt phosphorylation is a risk factor for early disease recurrence and poor prognosis in hepatocellular carcinoma. Cancer 103: 307-312, 2005.

26. Borlak J, Meier T, Halter R, Spanel R and Spanel-Borowski K: Epidermal growth factor-induced hepatocellular carcinoma: Gene expression profiles in precursor lesions, early stage and solitary tumours. Oncogene 24: 1809-1819, 2005. 
27. Höpfner M, Sutter AP, Huether A, Schuppan D, Zeitz M and Scherübl H: Targeting the epidermal growth factor receptor by gefitinib for treatment of hepatocellular carcinoma. J Hepatol 41: 1008-1016, 2004.

28. Shachaf CM, Kopelman AM, Arvanitis C, Karlsson A, Beer S, Mandl S, Bachmann MH, Borowsky AD, Ruebner B, Cardiff RD, et al: MYC inactivation uncovers pluripotent differentiation and tumour dormancy in hepatocellular cancer. Nature 431: 1112-1117, 2004.

29. Marrero JA and Lok AS: Newer markers for hepatocellular carcinoma. Gastroenterology 127 (5 Suppl 1): S113-S119, 2004.

30. Tovar V, Alsinet C, Villanueva A, Hoshida Y, Chiang DY, Solé M, Thung S, Moyano S, Toffanin S, Mínguez B, et al: IGF activation in a molecular subclass of hepatocellular carcinoma and pre-clinical efficacy of IGF-1R blockage. J Hepatol 52: 550-559, 2010.

31. Bekaii-Saab T, Williams N, Plass C, Calero MV and Eng C: A novel mutation in the tyrosine kinase domain of ERBB2 in hepatocellular carcinoma. BMC Cancer 6: 278, 2006.

32. Fornari F, Gramantieri L, Ferracin M, Veronese A, Sabbioni S Calin GA, Grazi GL, Giovannini C, Croce CM, Bolondi L and Negrini M: MiR-221 controls CDKN1C/p57 and CDKN1B/p27 expression in human hepatocellular carcinoma. Oncogene 27: $5651-5661,2008$.
33. Whittaker S, Marais $\mathrm{R}$ and Zhu AX: The role of signaling pathways in the development and treatment of hepatocellular carcinoma. Oncogene 29: 4989-5005, 2010.

34. Kerbel RS: Tumor angiogenesis. N Engl J Med 358: 2039-2049, 2008.

35. Kaseb AO, Hanbali A, Cotant M, Hassan MM, Wollner I and Philip PA: Vascular endothelial growth factor in the management of hepatocellular carcinoma: A review of literature. Cancer 115: 4895-4906, 2009.

36. Nischalke HD, Coenen M, Berger C, Aldenhoff K, Müller T, Berg T, Krämer B, Körner C, Odenthal M, Schulze F, et al: The toll-like receptor 2 (TLR2)-196 to $-174 \mathrm{del} /$ ins polymorphism affects viral loads and susceptibility to hepatocellular carcinoma in chronic hepatitis C. Int J Cancer 130: 1470-1475, 2012.

37. Timofeeva OA, Zhang X, Ressom HW, Varghese RS, Kallakury BV, Wang K, Ji Y, Cheema A, Jung M, Brown ML, et al: Enhanced expression of SOS1 is detected in prostate cancer epithelial cells from African-American men. Int $\mathrm{J}$ Oncol 35: 751-760, 2009.

38. De S, Dermawan JK and Stark GR: EGF receptor uses SOS1 to drive constitutive activation of NFKB in cancer cells. Proc Natl Acad Sci USA 111: 11721-11726, 2014. 